\title{
To Find Out the Suitable Time of Transplanting of Different Varieties of Basmati Rice with Respect to Yield
}

\author{
Nitin Maurya*, O.P. Singh, S.N. Singh, D.K. Tripati and Amit Kumar \\ Department of Crop Physiology C S Azad University of Agriculture and Technology \\ Kanpur-208002, India \\ *Corresponding author
}

\begin{tabular}{|c|}
\hline Keywords \\
\hline $\begin{array}{l}\text { Condition, Rice, } \\
\text { Transplanting, } \\
\text { Variety and yield }\end{array}$ \\
\hline Article Info \\
\hline $\begin{array}{l}\text { Accepted: } \\
12 \text { August } 2018 \\
\text { Available Online: } \\
\text { 10 September } 2018\end{array}$ \\
\hline
\end{tabular}

\section{Introduction}

Rice (Oryza sativa L.) is one of the most important cereal crops in Asia, grown under different hydrological conditions. About $90 \%$ of the world's rice is produced and consumed in Asia (FAO, 2014). It is the principal source of food for more than half of the world population (Nguyen et al., 2004), particularly respectively.

\section{A B S T R A C T}

The field experiment was conducted at Student's Instructional Farm, of Chandra Shekhar Azad University of Agriculture and Technology, Kanpur (U.P.), during two kharif season i.e., 2016 and 2017. The designed in split-plot design with three replication. The three dates of transplanting with., first date of transplanting $\left(03^{\text {st }}\right.$ July) $\mathrm{D}_{1}$, yield decreasing reduced second date of transplanting ( $16^{\text {st }}$ July) $\mathrm{D}_{2}$, and third transplanting $\left(30^{\text {st }} \mathrm{July}\right) \mathrm{D}_{3}$ conditions were allocated in the main plots and four basmati rice varieties PB-1 $\left(\mathrm{V}_{1}\right)$, PB$1509\left(\mathrm{~V}_{2}\right)$, PB-6 $\left(\mathrm{V}_{3}\right)$, and PB-1121 $\left(\mathrm{V}_{4}\right)$, in sub plot.. The significantly maximum mean value of first transplanting times to number of tiller found in the timely first transplanting date $\left(03^{\text {st } J u l y)} \mathrm{D}_{1}\right.$ i.e., 10.27 and 11.16 tiller hill $^{-1}$ at 30 DAT, 11.86 and 12.94 tiller hill $^{-1}$

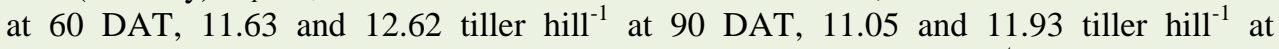
harvesting stage (DAT), while minimum in third transplanting date $\left(30^{\text {st }} \mathrm{July}\right) \mathrm{D}_{3}$ with 9.11 and 10.26 tiller hill ${ }^{-1}$ at $30 \mathrm{DAT}, 10.35$ and 11.33 tiller hill $^{-1}$ at $60 \mathrm{DAT}, 10.23$ and 11.86 tiller hill ${ }^{-1}$ at $90 \mathrm{DAT}, 9.61$ and 10.53 tiller hill $^{-1}$ at harvesting stage of concerning years respectively. Observed The mean value of varieties recorded significantly higher to number of tiller hill ${ }^{-1}$ with variety, (PB-1509) $\mathrm{V}_{2}$ (10.25 and 11.27 at 30 DAT, 12.62 and 13.17 at 60 DAT, 12.40 and 13.07 at 90 DAT, 11.60 and 12.62 tiller hill $^{-1}$ at harvesting DAT). Next to this were, variety, (PB-1121) $\mathrm{V}_{4}(9.82$ and $11.03 ; 11.49$ and $12.55 ; 11.66$ and $11.20 ; 10.80$ and 11.37 tiller hill $\left.^{-1}\right)$, variety, (PB-1) $\mathrm{V}_{1}(9.66$ and $10.29 ; 10.36$ and 11.69; 9.97 and 11.02; 9.59 and 10.71 tiller hill $^{-1}$ ) and lower in (PB-6) $\mathrm{V}_{3}$ (9.27 and 10.12; 9.79 and 11.01; 9.63 and 10.99; 9.21 and 10.24 tiller hill $^{-1}$ in year 2016 and 2017, in South and Southeast Asia, Latin America and Indonesia. In India, it is a dominating staple food crop of fertile and alluvial soils of North- West regions of the country, particularly Indo-Gangetic Plains (Walia and Walia, 2007). Rice contributes around $45 \%$ of the total production and being the staple food for more than two third of the population, holds the key to sustain food sufficiency in the 
country (Rai and Kushwaha, 2005). India is the second largest producer of rice in the world with production of 105.48 million tonnes from 43.90 million hectares, with a productivity of $2390 \mathrm{~kg} / \mathrm{ha}$ during 2015 (Economic survey, 2015-16)

\section{Materials and Method}

The present investigation entitled "Physiological studies on growth, development and yield component of Basmati rice under different planting times" was conducted at experiment Research Farm, Student's Instructional Farm, Chandra Shekhar Azad University of Agriculture and Technology, Kanpur (U.P.) during Kharif season i.e., 2016 and 2017 (Table 2).

\section{Location and climatic conditions}

Geographically Kanpur is located of $26.28^{\circ}$ North Longitude of $80.25^{\circ}$ East Longitude and above 127 meters sea level. It lies in the subtropical regions where rice is grown in Kharif seasons (Table 1).

\section{Fertilizer application}

Recommended doses of fertilizer were applied. Full dose of phosphorous (30 kg/ha) and $\mathrm{ZnSO} 4(25 \mathrm{~kg} / \mathrm{ha}$ ) was applied at the time of preparatory tillage by broadcasting under transplanted plots. Nitrogen $(60 \mathrm{~kg} / \mathrm{ha})$ was applied in three split doses i.e. 1/3rd at transplanting, $1 / 3 \mathrm{rd}$ at 21 days after transplanting (DAT) and remaining 1/3rd at 42 DAT in transplanted plots.

\section{Irrigation schedule}

Field was irrigated frequently so as to maintain the $5 \mathrm{~cm}$ level of standing water till 15 days after transplanting. Thereafter irrigation was given as and when required to maintain the saturated conditions of soil.

\section{Results and Discussion}

\section{Numbers of tillers hill $^{-1}$}

Number of tillers hill ${ }^{-1}$ under each treatment was recorded by visual counting at the appropriate stage. The plants already tagged for this purpose was used and average tillers hill $^{-1}$ were counting at different stages. The data stated in table 3(A), (B) and (D), for number of tiller $\left(\right.$ hill $\left.^{-1}\right)$ as affected by transplanting times, basmati rice varieties, treatments and their interaction effects at 30, 60 and 90 and Harvesting stage (DAT).

\section{Effect of transplanting dates}

The significantly maximum mean value of first transplanting times to number of tiller found in the timely first transplanting time $\left(\mathrm{D}_{1}\right)$ i.e., 10.27 and 11.16 tiller hill $^{-1}$ at 30 DAT, 11.86 and 12.94 tiller hill $^{-1}$ at 60 DAT, 11.63 and 12.62 tiller hill $^{-1}$ at $90 \mathrm{DAT}, 11.05$

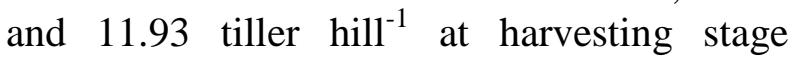
(DAT), while minimum in third transplanting date $\left(D_{3}\right)$ with 9.11 and 10.26 tiller hill ${ }^{-1}$ at 30 DAT, 10.35 and 11.33tiller hill ${ }^{-1}$ at 60 DAT, 10.23 and 11.86 tiller hill $^{-1}$ at 90 DAT, 9.61 and 10.53 tiller hill $^{-1}$ at harvesting stage of concerning years respectively (Table 4).

\section{Effect of varieties}

The mean value of varieties recorded significantly higher to number of tiller hill $^{-1}$ with variety $\mathrm{V}_{2}(10.25$ and 11.27 at 30 DAT, 12.62 and 13.17 at 60 DAT, 12.40 and 13.07 at 90 DAT, 11.60 and 12.62tiller hill $^{-1}$ at harvesting DAT). Next to this were, variety $\mathrm{V}_{4}$ (9.82 and $11.03 ; 11.49$ and $12.55 ; 11.66$ and $11.20 ; 10.80$ and 11.37 tiller hill $^{-1}$ ), variety $\mathrm{V}_{1}$ (9.66 and $10.29 ; 10.36$ and $11.69 ; 9.97$ and 11.02; 9.59 and 10.71 tiller hill $^{-1}$ ) and lower in $\mathrm{V}_{3}(9.27$ and $10.12 ; 9.79$ and $11.01 ; 9.63$ and 10.99; 9.21 and 10.24tiller hill $^{-1}$ in year 2016 and 2017 , respectively. 
Table.1 Weather parameters prevailed during experimental year 2016 at experimental farm, Kanpur

\begin{tabular}{|c|c|c|c|c|c|c|}
\hline \multirow[t]{2}{*}{ SMW } & \multirow[t]{2}{*}{ Date to Date } & \multicolumn{2}{|c|}{ Temperature(C) } & \multicolumn{2}{|c|}{ Relative humidity (\%) } & \multirow{2}{*}{$\begin{array}{l}\text { Rain Fall } \\
(\mathrm{mm})\end{array}$} \\
\hline & & Max. & Min. & Max. & Min. & \\
\hline 1 & $02.07 .2016-08.07 .2016$ & 31.6 & 25.1 & 92 & 81 & 126.1 \\
\hline 2 & $09.07 .2016-15.07 .2016$ & 32.8 & 26.1 & 89 & 82 & 77.6 \\
\hline 3 & $16.07 .2016-22.07 .2016$ & 31.7 & 26.1 & 88 & 81 & 30.2 \\
\hline 4 & $23.07 .2016-29.07 .2016$ & 32.6 & 25.9 & 90 & 76 & 29.9 \\
\hline 5 & $30.07 .2016-05.08 .2016$ & 32.1 & 25.6 & 91.1 & 74.7 & 70.7 \\
\hline 6 & $06.08 .2016-12.08 .2016$ & 32.3 & 26.2 & 89 & 74 & 67.9 \\
\hline 7 & $13.08 .2016-19.08 .2016$ & 31.7 & 25.5 & 92 & 77 & 33.1 \\
\hline 8 & $20.08 .2016-26.08 .2016$ & 32.7 & 25.2 & 85 & 70 & 5.6 \\
\hline 9 & $27.08 .2016-02.09 .2016$ & 34.3 & 26.5 & 87 & 68 & 8.4 \\
\hline 10 & $03.09 .2016-09.09 .2016$ & 33.8 & 25.9 & 82 & 64 & 00 \\
\hline 11 & $10.09 .2016-16.09 .2016$ & 32.8 & 25.0 & 87 & 69 & 7.0 \\
\hline 12 & $17.09 .2016-23.09 .2016$ & 32.6 & 25.3 & 89 & 76 & 7.8 \\
\hline 13 & $24.09 .2016-30.09 .2016$ & 32.6 & 24.1 & 92 & 66 & 00 \\
\hline 14 & $01.10 .2016-07.10 .2016$ & 34.9 & 24.8 & 84 & 59 & 20.0 \\
\hline 15 & $08.10 .2016-14.10 .2016$ & 33.5 & 21.4 & 84 & 57 & 14.0 \\
\hline 16 & $15.10 .2016-21.10 .2016$ & 33.7 & 16.6 & 84 & 38 & 00 \\
\hline 17 & $22.10 .2016-28.10 .2016$ & 33.7 & 16.6 & 77 & 35 & 00 \\
\hline 18 & $29.10 .2016-04.11 .2016$ & 31.5 & 14.0 & 89 & 38 & 00 \\
\hline 19 & $05.11 .2016-11.11 .2016$ & 30.3 & 13.2 & 83 & 42 & 00 \\
\hline 20 & $12.11 .2016-18.11 .2016$ & 29.2 & 12.1 & 86 & 42 & 00 \\
\hline 21 & $19.11 .2016-25.11 .2016$ & 28.8 & 11.8 & 81 & 42 & 00 \\
\hline 22 & $26.11 .2016-02.12 .2016$ & 25.6 & 13.1 & 87 & 62 & 00 \\
\hline
\end{tabular}

Table.2 Experimental details

\begin{tabular}{|ll|}
\hline- & Location \\
\hline- & Statistical Design \\
\hline- & Replication \\
\hline$-\quad$ & Single Plot Size \\
\hline- & Total number of plots \\
\hline- & Date of transplanting \\
\hline- & Varieties \\
\hline - & Plant to plant distance \\
\hline - & Row to row distance \\
\hline - & Detail about treatment \\
\hline Dates of transplanting (D) \\
\hline D $_{1:}$ First date transplanting $\mathbf{0 3}^{\text {st }} \mathbf{J u l y}$ \\
\hline D $_{2:}$ Second date transplanting $\mathbf{1 6}^{\text {st }} \mathbf{J u l y}$ \\
\hline D $_{3:}$ Third date transplanting30 \\
\hline
\end{tabular}

Student's Instructional Farm, Kanpur

: $\quad$ Spilt-plot Design

Main plot: Date of transplanting (D)

Sub plot: Variety (V)

: $\quad$ Three (3)

: $4 \times 4 \mathrm{~m}^{2}$

: 36

: 3 (Both year on same date)

: 4

: $10 \mathrm{~cm}$

: $\quad 20 \mathrm{~cm}$

:

: $\quad$ Varieties (V)

: $\mathrm{V}_{1:} \mathrm{PB}-1$

: $\quad \mathrm{V}_{2}: \mathrm{PB}-1509$

: $\quad \mathrm{V}_{3}$ : $\mathrm{PB}-6$

$\mathrm{V}_{4}$ : PB-1121 
Table.3 (A) Effect of different transplanting times on number of tiller hill ${ }^{-1}$ at 30 (DAT) of Basmati rice varieties

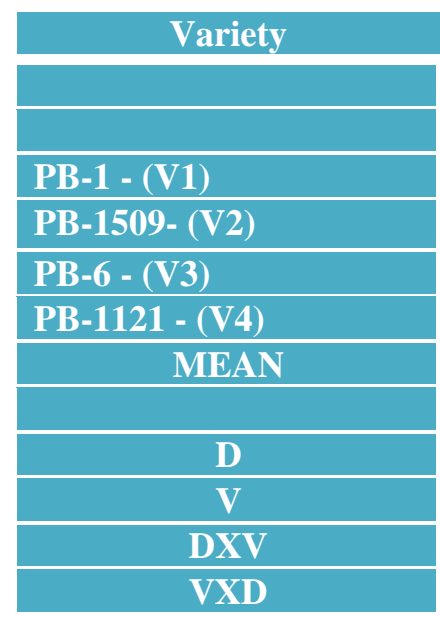

\begin{tabular}{|c|c|c|c|}
\hline \multicolumn{4}{|c|}{ Number of tiller hill ${ }^{-1} 30 \mathrm{DAT}$} \\
\hline \multicolumn{4}{|c|}{2016} \\
\hline D1 & D2 & D3 & MEAN \\
\hline 10.14 & 9.83 & 9.01 & 9.66 \\
\hline 10.81 & 10.03 & 9.93 & 10.25 \\
\hline 9.95 & 9.63 & 8.25 & 9.27 \\
\hline 10.18 & 10.00 & 9.28 & 9.82 \\
\hline 10.27 & 9.87 & 9.11 & \\
\hline \multicolumn{2}{|c|}{ SE (dff) } & \multicolumn{2}{|c|}{$\mathrm{CD}(5 \%)$} \\
\hline \multicolumn{2}{|c|}{0.08} & \multicolumn{2}{|c|}{0.24} \\
\hline \multicolumn{2}{|c|}{0.06} & \multicolumn{2}{|c|}{0.14} \\
\hline \multicolumn{2}{|c|}{0.11} & \multicolumn{2}{|c|}{0.24} \\
\hline \multicolumn{2}{|c|}{0.12} & \multicolumn{2}{|c|}{025} \\
\hline
\end{tabular}

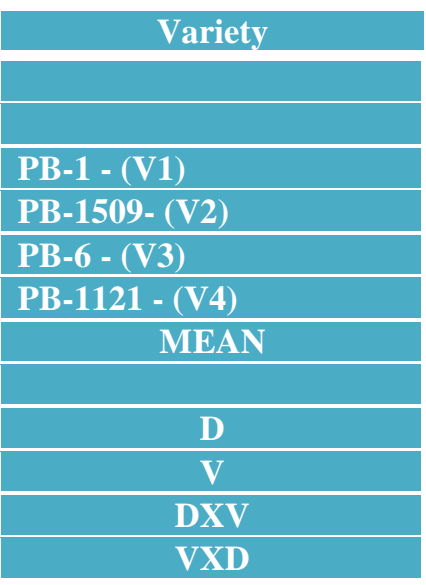

\begin{tabular}{|c|c|c|c|}
\hline \multicolumn{4}{|c|}{ Number of tiller hill ${ }^{-1}$ 30DAT } \\
\hline \multicolumn{4}{|c|}{2017} \\
\hline D1 & D2 & D3 & MEAN \\
\hline 10.47 & 10.18 & 10.23 & 10.29 \\
\hline 12.01 & 11.23 & 10.57 & 11.27 \\
\hline 10.33 & 10.03 & 10.01 & 10.12 \\
\hline 11.85 & 11.01 & 10.25 & 11.03 \\
\hline 11.16 & 10.61 & 10.26 & \\
\hline \multicolumn{2}{|c|}{ SE (dff) } & \multicolumn{2}{|c|}{$\mathrm{CD}(5 \%)$} \\
\hline \multicolumn{2}{|c|}{0.06} & \multicolumn{2}{|c|}{0.16} \\
\hline \multicolumn{2}{|c|}{0.06} & \multicolumn{2}{|c|}{0.13} \\
\hline \multicolumn{2}{|c|}{0.11} & \multicolumn{2}{|c|}{0.23} \\
\hline \multicolumn{2}{|c|}{0.13} & \multicolumn{2}{|c|}{0.27} \\
\hline
\end{tabular}

Table.3 (B) Effect of different transplanting times on number of tiller hill ${ }^{-1}$ at 60 (DAT) of Basmati rice varieties

\begin{tabular}{|c|}
\hline Variety \\
\hline \\
\hline \\
\hline PB-1 - (V1) \\
\hline PB-1509- (V2) \\
\hline PB-6 - (V3) \\
\hline PB-1121 - (V4) \\
\hline MEAN \\
\hline \\
\hline D \\
\hline V \\
\hline DXV \\
\hline VXD \\
\hline
\end{tabular}

\begin{tabular}{|c|c|c|c|}
\hline \multicolumn{4}{|c|}{ Number of tiller hill $^{-1}$ 60DAT } \\
\hline \multicolumn{4}{|c|}{2016} \\
\hline D1 & D2 & D3 & MEAN \\
\hline 10.88 & 10.18 & 10.03 & 10.36 \\
\hline 14.03 & 12.63 & 11.22 & 12.62 \\
\hline 10.06 & 9.92 & 9.39 & 9.79 \\
\hline 12.50 & 11.20 & 10.78 & 11.49 \\
\hline 11.86 & 10.98 & 10.35 & \\
\hline \multicolumn{2}{|c|}{ SE (dff) } & \multicolumn{2}{|c|}{$\mathrm{CD}(5 \%)$} \\
\hline \multicolumn{2}{|c|}{0.14} & \multicolumn{2}{|c|}{0.41} \\
\hline \multicolumn{2}{|c|}{0.07} & \multicolumn{2}{|c|}{0.15} \\
\hline \multicolumn{2}{|c|}{0.12} & \multicolumn{2}{|c|}{0.26} \\
\hline \multicolumn{2}{|c|}{0.16} & \multicolumn{2}{|c|}{0.33} \\
\hline
\end{tabular}


Int.J.Curr.Microbiol.App.Sci (2018) 7(9): 1728-1736

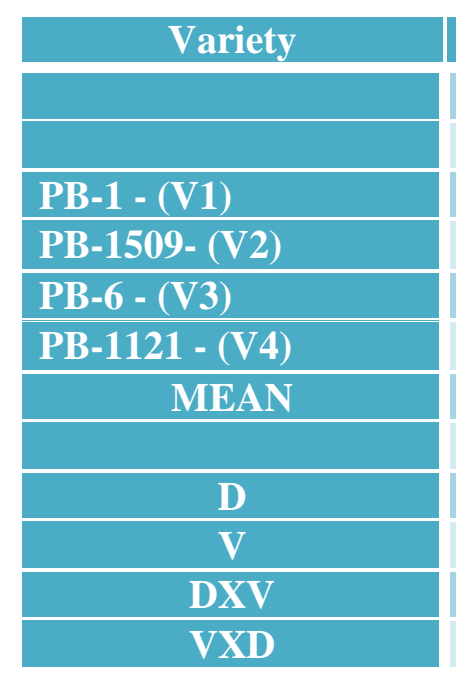

\begin{tabular}{|c|c|c|c|}
\hline \multicolumn{4}{|c|}{ Number of tiller hill ${ }^{-1} 60 \mathrm{DAT}$} \\
\hline \multicolumn{4}{|c|}{2017} \\
\hline D1 & D2 & D3 & MEAN \\
\hline 12.51 & 11.35 & 11.21 & 11.69 \\
\hline 14.23 & 13.27 & 12.02 & 13.17 \\
\hline 11.28 & 11.17 & 10.58 & 11.01 \\
\hline 13.75 & 12.39 & 11.51 & 12.55 \\
\hline 12.94 & 12.04 & 11.33 & \\
\hline \multicolumn{2}{|c|}{$\mathrm{SE}(\mathrm{dff})$} & \multicolumn{2}{|c|}{$\mathrm{CD}(5 \%)$} \\
\hline \multicolumn{2}{|c|}{0.17} & \multicolumn{2}{|c|}{0.48} \\
\hline \multicolumn{2}{|c|}{0.08} & \multicolumn{2}{|c|}{0.17} \\
\hline \multicolumn{2}{|c|}{0.14} & \multicolumn{2}{|c|}{0.30} \\
\hline \multicolumn{2}{|c|}{0.18} & \multicolumn{2}{|c|}{0.38} \\
\hline
\end{tabular}

Table.3 (C) Effect of different transplanting times on number of tiller hill ${ }^{-1}$ at 90 (DAT) of Basmati rice varieties

\begin{tabular}{|c|}
\hline Variety \\
\hline \\
\hline \\
\hline PB-1 - (V1) \\
\hline PB-1509- (V2) \\
\hline PB-6 - (V3) \\
\hline PB-1121 - (V4) \\
\hline MEAN \\
\hline \\
\hline D \\
\hline V \\
\hline DXV \\
\hline VXD \\
\hline
\end{tabular}

\begin{tabular}{|c|c|c|c|}
\hline \multicolumn{4}{|c|}{ Number of tiller hill ${ }^{-1}$ 90DAT } \\
\hline \multicolumn{4}{|c|}{2016} \\
\hline D1 & D2 & D3 & MEAN \\
\hline 10.71 & 9.85 & 9.35 & 9.97 \\
\hline 13.30 & 12.42 & 11.47 & 12.40 \\
\hline 10.03 & 9.75 & 9.12 & 9.63 \\
\hline 12.50 & 11.48 & 11.00 & 11.66 \\
\hline 11.63 & 10.87 & 10.23 & \\
\hline \multicolumn{2}{|c|}{ SE (dff) } & \multicolumn{2}{|c|}{$\mathrm{CD}(5 \%)$} \\
\hline \multicolumn{2}{|c|}{0.19} & \multicolumn{2}{|c|}{0.54} \\
\hline \multicolumn{2}{|c|}{0.13} & \multicolumn{2}{|c|}{0.27} \\
\hline \multicolumn{2}{|c|}{0.23} & \multicolumn{2}{|c|}{ N.S. } \\
\hline \multicolumn{2}{|c|}{0.26} & \multicolumn{2}{|c|}{ N.S. } \\
\hline
\end{tabular}

\begin{tabular}{|c|}
\hline Variety \\
\hline \\
\hline \\
\hline PB-1 - (V1) \\
\hline PB-1509- (V2) \\
\hline PB-6 - (V3) \\
\hline PB-1121 - (V4) \\
\hline MEAN \\
\hline \\
\hline D \\
\hline V \\
\hline DXV \\
\hline VXD \\
\hline
\end{tabular}

\begin{tabular}{|c|c|c|c|}
\hline \multicolumn{4}{|c|}{ Number of tiller hill ${ }^{-1}$ 90DAT } \\
\hline \multicolumn{4}{|c|}{2017} \\
\hline D1 & D2 & D3 & MEAN \\
\hline 12.01 & 11.02 & 11.02 & 11.02 \\
\hline 14.10 & 13.07 & 13.07 & 13.07 \\
\hline 11.12 & 11.00 & 11.00 & 10.99 \\
\hline 13.26 & 12.35 & 12.35 & 11.20 \\
\hline 12.62 & 11.86 & 11.86 & 11.57 \\
\hline \multicolumn{2}{|c|}{ SE (dff) } & \multicolumn{2}{|c|}{$\mathrm{CD}(5 \%)$} \\
\hline \multicolumn{2}{|c|}{0.21} & \multicolumn{2}{|c|}{0.58} \\
\hline \multicolumn{2}{|c|}{0.10} & \multicolumn{2}{|c|}{0.21} \\
\hline \multicolumn{2}{|c|}{0.17} & \multicolumn{2}{|c|}{0.36} \\
\hline \multicolumn{2}{|c|}{0.20} & \multicolumn{2}{|c|}{0.44} \\
\hline
\end{tabular}


Table.4 Effect of different transplanting times on number of tiller hill ${ }^{-1}$ at Harvesting (DAT) of Basmati rice varieties

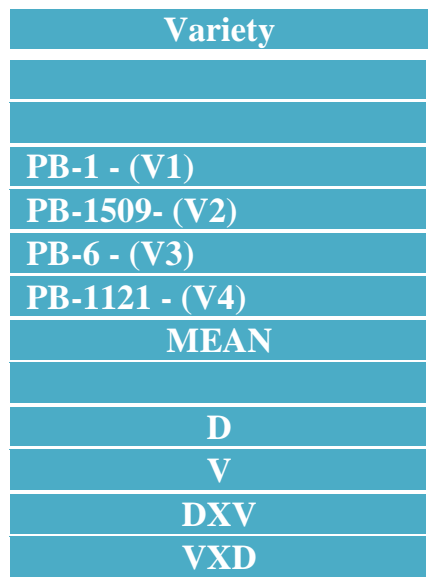

Variety

\begin{tabular}{|c|}
\hline \\
\hline PB-1 - (V1) \\
\hline PB-1509- (V2) \\
\hline PB-6 - (V3) \\
\hline PB-1121 - (V4) \\
\hline MEAN \\
\hline D \\
\hline V \\
\hline DXV \\
\hline VXD \\
\hline
\end{tabular}

Number of tiller hill ${ }^{-1}$ harvesting DAT

\begin{tabular}{|c|c|c|c|}
\hline \multicolumn{4}{|c|}{2016} \\
\hline D1 & D2 & D3 & MEAN \\
\hline 9.84 & 9.72 & 9.21 & 9.59 \\
\hline 12.50 & 11.95 & 10.36 & 11.60 \\
\hline 9.63 & 9.21 & 8.80 & 9.21 \\
\hline 12.25 & 10.09 & 10.07 & 10.80 \\
\hline 11.05 & 10.24 & 9.61 & \\
\hline \multicolumn{2}{|c|}{$\mathrm{SE}(\mathrm{dff})$} & \multicolumn{2}{|c|}{$\mathrm{CD}(5 \%)$} \\
\hline \multicolumn{2}{|c|}{0.09} & \multicolumn{2}{|c|}{0.26} \\
\hline \multicolumn{2}{|c|}{0.11} & \multicolumn{2}{|c|}{0.24} \\
\hline \multicolumn{2}{|c|}{0.20} & \multicolumn{2}{|c|}{0.42} \\
\hline \multicolumn{2}{|c|}{0.18} & \multicolumn{2}{|c|}{0.36} \\
\hline
\end{tabular}

Table.5 Effect of different transplanting times on Panicle length plant ${ }^{-1}(\mathrm{~cm})$ after harvesting of Basmati rice varieties

\begin{tabular}{|c|}
\hline \multicolumn{1}{|c|}{ Variety } \\
\hline \\
\hline PB-1 - (V1) \\
\hline PB-1509- (V2) \\
\hline PB-6 - (V3) \\
\hline PB-1121 - (V4) \\
\hline MEAN \\
\hline \\
\hline D \\
\hline V \\
\hline DXV \\
\hline VXD \\
\hline
\end{tabular}

\begin{tabular}{|c|c|c|c|}
\hline \multicolumn{4}{|c|}{$\begin{array}{r}\text { Number of tiller hill }{ }^{-1} h \\
2017\end{array}$} \\
\hline D1 & D2 & D3 & MEAN \\
\hline 11.13 & 10.89 & 10.12 & 10.71 \\
\hline 13.76 & 12.60 & 11.50 & 12.62 \\
\hline 10.51 & 10.20 & 10.03 & 10.24 \\
\hline 12.34 & 11.29 & 10.50 & 11.37 \\
\hline 11.93 & 11.24 & 10.53 & \\
\hline \multicolumn{2}{|c|}{ SE (dff) } & \multicolumn{2}{|c|}{$\mathrm{CD}(5 \%)$} \\
\hline \multicolumn{2}{|c|}{0.14} & \multicolumn{2}{|c|}{0.39} \\
\hline \multicolumn{2}{|c|}{0.07} & \multicolumn{2}{|c|}{0.15} \\
\hline \multicolumn{2}{|c|}{0.12} & \multicolumn{2}{|c|}{0.26} \\
\hline \multicolumn{2}{|c|}{0.20} & \multicolumn{2}{|c|}{0.42} \\
\hline
\end{tabular}


Int.J.Curr.Microbiol.App.Sci (2018) 7(9): 1728-1736

\begin{tabular}{|c|c|c|c|c|}
\hline Variety & \multicolumn{4}{|c|}{ Panicle length plant ${ }^{-1}(\mathrm{~cm})$} \\
\hline & \multicolumn{4}{|c|}{2017} \\
\hline & D1 & D2 & D3 & MEAN \\
\hline PB-1 - (V1) & 24.09 & 22.62 & 20.81 & 25.50 \\
\hline PB-1509- (V2) & 27.30 & 25.30 & 24.87 & 25.82 \\
\hline PB-6 - (V3) & 23.73 & 21.51 & 18.79 & 21.34 \\
\hline PB-1121 - (V4) & 25.93 & 23.87 & 22.22 & 24.00 \\
\hline \multirow[t]{2}{*}{ MEAN } & 25.26 & 23.32 & 21.67 & \\
\hline & \multicolumn{2}{|c|}{ SE (dff) } & \multicolumn{2}{|c|}{$\mathrm{CD}(5 \%)$} \\
\hline$\overline{\mathbf{D}}$ & \multicolumn{2}{|c|}{0.75} & \multicolumn{2}{|c|}{2.09} \\
\hline $\mathbf{V}$ & \multicolumn{2}{|c|}{0.72} & \multicolumn{2}{|c|}{1.52} \\
\hline DXV & \multicolumn{2}{|c|}{1.25} & \multicolumn{2}{|c|}{ N.S. } \\
\hline VXD & \multicolumn{2}{|c|}{1.28} & \multicolumn{2}{|c|}{ N.S. } \\
\hline
\end{tabular}

Table.6 Effect of different transplanting times on Number of grains/hill after harvesting of Basmati rice varieties

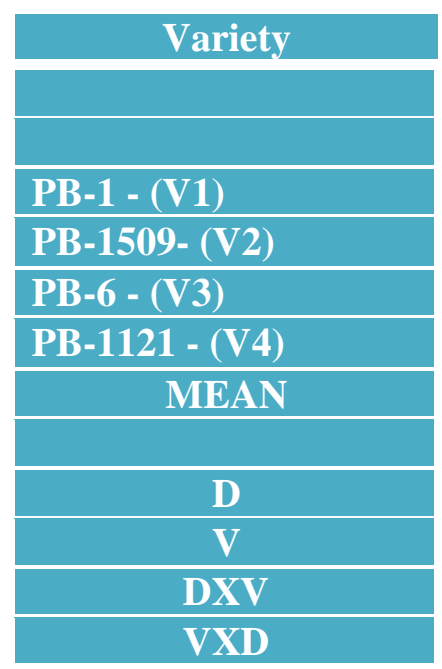

\section{Variety}

\begin{tabular}{|c|}
\hline \\
\hline PB-1 - (V1) \\
\hline PB-1509- (V2) \\
\hline PB-6 - (V3) \\
\hline PB-1121 - (V4) \\
\hline MEAN \\
\hline D \\
\hline V \\
\hline DXV \\
\hline VXD \\
\hline
\end{tabular}

\begin{tabular}{|c|c|c|c|}
\hline \multicolumn{4}{|c|}{ Number of grains/hill } \\
\hline \multicolumn{4}{|c|}{2016} \\
\hline D1 & D2 & D3 & MEAN \\
\hline 355.49 & 335.11 & 293.56 & 328.05 \\
\hline 433.20 & 396.25 & 352.40 & 393.95 \\
\hline 340.26 & 304.31 & 251.17 & 298.58 \\
\hline 388.50 & 339.37 & 299.10 & 342.32 \\
\hline 379.36 & 343.76 & 299.05 & \\
\hline \multicolumn{2}{|c|}{ SE $($ dff $)$} & \multicolumn{2}{|c|}{$\mathrm{CD}(5 \%)$} \\
\hline \multicolumn{2}{|c|}{7.39} & \multicolumn{2}{|c|}{20.40} \\
\hline \multicolumn{2}{|c|}{7.58} & \multicolumn{2}{|c|}{15.93} \\
\hline \multicolumn{2}{|c|}{13.13} & \multicolumn{2}{|c|}{ N.S. } \\
\hline \multicolumn{2}{|c|}{14.91} & \multicolumn{2}{|c|}{ N.S. } \\
\hline
\end{tabular}

\begin{tabular}{|c|c|c|c|}
\hline \multicolumn{4}{|c|}{ Number of grains/hill } \\
\hline \multicolumn{4}{|c|}{2017} \\
\hline D1 & D2 & D3 & MEAN \\
\hline 362.44 & 341.24 & 300.56 & 334.74 \\
\hline 442.14 & 404.80 & 361.41 & 402.78 \\
\hline 349.15 & 312.85 & 260.40 & 307.46 \\
\hline 400.81 & 350.37 & 310.03 & 353.73 \\
\hline 388.63 & 352.31 & 308.10 & \\
\hline \multicolumn{2}{|c|}{ SE (dff) } & \multicolumn{2}{|c|}{$\mathrm{CD}(5 \%)$} \\
\hline \multicolumn{2}{|c|}{9.23} & \multicolumn{2}{|c|}{25.50} \\
\hline \multicolumn{2}{|c|}{9.49} & \multicolumn{2}{|c|}{19.94} \\
\hline \multicolumn{2}{|c|}{16.44} & \multicolumn{2}{|c|}{ N.S. } \\
\hline \multicolumn{2}{|c|}{18.55} & \multicolumn{2}{|c|}{ N.S. } \\
\hline
\end{tabular}




\section{Panicle length plant $^{-1}(\mathrm{~cm})$}

The data elucidated for Panicle length plant ${ }^{-1}$ (cm) as affected by different transplanting times on basmati rice varieties and their interaction on have been presented in Table 5 .

\section{Effect of transplanting dates}

It the mean value of transplanting times Panicle length plant $^{-1}$ in $\mathrm{cm}$ of different transplanting dates significantly influence the panicle length plant ${ }^{-1}$ accumulation in $(\mathrm{cm})$. The statistically maximum panicle length plant $^{-1}(24.50$ and $25.26 \mathrm{~cm})$ was recorded first transplanting date $\left(\mathrm{D}_{1}\right)$ and minimum $(20.88$ and $21.67 \mathrm{~cm}$ ) with $\mathrm{D}_{3}$ during 2016 and 2017 respectively.

\section{Effect of varieties}

Significant effect of varieties was found for the accumulation of Panicle length plant ${ }^{-1}$. Among the varieties, $\mathrm{V}_{2}$ was recorded significantly superior $(25.08 \& 25.82 \mathrm{~cm})$ followed by $\mathrm{V}_{4}(23.10 \& 24.00 \mathrm{~cm}), \mathrm{V}_{1}(21.70$ $\& 25.50 \mathrm{~cm})$ and mean while minimum in $(20.64 \& 21.34 \mathrm{~cm})$ in year 2016 and 2017 , respectively.

\section{Number of grains hill ${ }^{-1}$}

The data elucidated for Number of grains hill $^{-1}$ as affected by different transplanting times on basmati rice varieties, and their interaction on have been presented in Table 6 .

\section{Effect of transplanting dates}

It is visualized from the mean value of different transplanting dates significantly influence the number of grains accumulation in $\left(\right.$ hill $\left.^{-1}\right)$. The statistically maximum number of grains hill ${ }^{-1}$ (379.36 and 388.63hill ${ }^{-1}$ ) was recorded first transplanting date $\left(\mathrm{D}_{1}\right)$ and minimum (299.05 and 308.10hill ${ }^{-1}$ ) with $\mathrm{D}_{3}$ during 2016 and 2017 respectively.

\section{Effect of varieties}

Significant effect of varieties was found for the accumulation of number of grains. Among the varieties, $\mathrm{V}_{2}$ was recorded significantly superior (393.95 \& 402.78 hill $^{-1}$ ) followed by $\mathrm{V}_{4}\left(342.32 \&\right.$ 353.73hill $\left.{ }^{-1}\right), \mathrm{V}_{1}(328.05 \&$ 334.74hill ${ }^{-1}$ ) and mean while minimum in $\left(298.58 \& 307.46\right.$ hill $\left.^{-1}\right)$ in year 2016 and 2017 , respectively.

\section{References}

Akbar, N., Iqbal, A., Khan, H.Z., Hanif, M.K. and Bashir, M.U. (2010). Effect of different sowing dates on the yield and yield components of direct seeded fine rice (Oryza sativa L.). J. of Plant Breeding and Crop Sci., 2(10): 312-315.

Akram, M.; Cheema, A.A.; Awn, M.A. and Maqbool, A. (1985). Effect of planting date and fertilizer level on grain yield and protein content of rice. Pak. J. Agric. Res., 6 (3): 165-167.

Baloch, M.S., Ullah, A.I., Gul, H. (2006). Growth and yield of rice as affected by transplanting dates and seedlings per hill under high temperature of Dera Ismail Khan. Pakistan J. Zhejiang Univ. Sci., 7(7): 572-579.

Canet, R. (1986). Effect of sowing time on yield of rice varieties in two locations in Cuba. Intl. Rice Res. Newsl. 11(3): 28.

Dawadi, K.P., and Chaudhary, N.K. (2013). Effect of sowing dates and varieties on yield and yield attributes of direct seeded rice in Chitwan, Nepal. Internat. J. Agril. Sci Res., 2: 95-102.

Dhiman, S.D.; Om, H.; Singh, B. and Bikram, Singh (1995). Yield stabilization of scented dwarf rice under late planting through spacing and seedling age. Haryana Agril. Univ. J. Res., 25 (3): 9599.

Khalifa Ali and Abou, A.B. (2009a). Evaluation of some hybrid rice varieties 
under different sowing times. Afri. J. Pl. Sci. 3(4): 53-58.

Koyamma, T., Kali, S.E. and Chammek, C. (1973).Effect of transplanting time on rice plant growth and grain yield in Thailand. Tech. Bul. Trop. Agric Res. Centre., 5: 32.
Mazid, A. and Ahmad, S. (1975). Effect of transplanting date on paddy yield and other plant characters in deferent rice varieties. J. Agric. Res., 13 (2): 447454.

\section{How to cite this article:}

Nitin Maurya, O.P. Singh, S.N. Singh, D.K. Tripati and Amit Kumar. 2018. To Find Out the Suitable Time of Transplanting of Different Varieties of Basmati Rice with Respect to Yield. Int.J.Curr.Microbiol.App.Sci. 7(09): 1728-1736. doi: https://doi.org/10.20546/ijcmas.2018.709.209 